\title{
Focusing on a complete blood cell parameter: mean platelet volume levels may be a predictor of coronary slow flow
}

This article was published in the following Dove Press journal:

Vascular Health and Risk Management

7 July 2017

Number of times this article has been viewed

\author{
Mücahid Yılmaz' \\ Mustafa Necati Dağ|ı \\ Ökkeș Uku' \\ Mehmet Nail Bilen' \\ Hasan Korkmaz ${ }^{2}$ \\ Kenan Erdem ${ }^{3}$ \\ Ertuğrul Kurtoğlu' \\ 'Department of Cardiology, Elazı̆̆ \\ Education and Research Hospital, \\ ${ }^{2}$ Department of Cardiology, FIRAT \\ University School of Medicine, Elazığ, \\ ${ }^{3}$ Department of Cardiology, Sivas \\ Hospital State, Sivas, Turkey
}

Background: The relationship between increased mean platelet volume (MPV) and atherosclerosis is well known. In the present study, MPV in patients with coronary slow flow (CSF) and in cases with normal coronary anatomy (NCA) was investigated and compared with the aim of identifying the relationship between CSF and MPV.

Patients and methods: We studied 40 patients previously determined via coronary angiography as having NCA and 40 patients with CSF in the coronary blood stream, as identified by thrombolysis in myocardial infarction square. Thus, a total of 80 patients from the Elaziğ Education and Research Hospital (Elaziğ, Turkey) were included in the present study retrospectively and laboratory and anamnesis information was scanned into their files. The relationship between MPV and CSF was studied. Results: MPV levels were observed to be significantly higher in the CSF group compared to the NCA group $(10.05 \pm 1.3$ and $8.6 \pm 0.6, p<0.001)$. In receiver operating characteristics analyses, it was determined that an MPV $>9.05$ measured in CSF patients at application had a predictive specificity of $77.5 \%$ and sensitivity of $77.5 \%$ for CSF (area under the curve: $0.825,95 \%$ confidence interval $[\mathrm{CI}]: 0.726-0.924, p<0.0001)$. It was found that MPV level was an independent predictor of CSF ( $\beta=-600, p<0.001,95 \% \mathrm{CI}$ : -0.383 to -0.176$)$.

Conclusion: MPV is increased in patients with CSF when compared to patients with NCA. This finding supports the fact that MPV could be a predictor of CSF.

Keywords: coronary slow flow, mean platelet volume, atherosclerosis

\section{Introduction}

The slow flow phenomenon is defined as a microvascular disorder characterized by slow progression of the opaque substance given during coronary angiography (CAG) toward distal vascular structures without any obstruction in epicardial coronary arteries. ${ }^{1}$ Initially, coronary slow flow (CSF) was defined in 1972 and it was claimed that this phenomenon could be induced by anomalies in coronary microcirculation. ${ }^{2}$ Left and right ventricle biopsies in patients with CSF demonstrate thickening in the capillary endothelium, constriction of the lumen, depletion of normal morphology of the nucleus, and histopathological symptoms of capillary vein diseases such as pycnosis., ${ }^{3,4}$ Thus, CSF is considered to be a variant of coronary heart disease.

Platelets play a significant role in the formation and progress of atherosclerotic coronary lesions. In empirical atherosclerotic coronary disease models, pre-administration of antiplatelet agents could prevent atherosclerosis development. It has been shown that platelets are more active in patients with acute coronary syndrome compared to healthy controls. ${ }^{5,6}$
Correspondence: Mücahid Yılmaz, Department of Cardiology, Elazığ Education and Research Hospital, Rızaiye Neighbourhood, İnönü Street 74, 23200 Center/Elazığ, Turkey

Tel +904242381000

Email mucahid.yilmaz@mynet.com 
The mean platelet volume (MPV) test is an indicator of the size of platelets. An increase in MPV value indicates an increase in platelet volume. ${ }^{7}$ Changes in the volumetric parameters of platelets could have prophylactic or diagnostic significance in thrombotic and pre-thrombotic events. ${ }^{8}$ Large platelets are more active enzymatically and metabolically than small platelets and produce more thromboxane A2 (TxA2). ${ }^{9}$ MPV is a physiological variable with hemostatic significance and an indicator of platelet activation. An increase in platelet volume has been reported in acute myocardial infarction, acute cerebral ischemia, and transient ischemic attacks. ${ }^{10}$ It has also been demonstrated that MPV could be an indicator of platelet activation, which plays an important role in atherosclerosis physiopathology. ${ }^{11}$ Increased MPV values are considered to closely correlate with primarily acute coronary syndromes and cardiovascular diseases, as well as well-known risk factors such as hypertension (HT), diabetes mellitus (DM), and hyperlipidemia (HL). ${ }^{12-15}$

MPV, an indicator of platelet activation, has an independent effect on the pathophysiology of atherosclerosis in the presence of other risk factors. ${ }^{16}$ It is one of the platelet function indices that reflects platelet production rate and platelet stimulation. Elevated MPV levels are reported in cardiovascular diseases. ${ }^{17}$ However, as there are few data regarding the relationship between MPV and CSF, this study was designed to compare the MPV levels of patients with CSF and that of normal controls. Are MPV levels high in CSF? What is the correlation between CSF and MPV? The present study scrutinizes the correlation between MPV and CSF, a variant of coronary heart disease, and determines whether MPV is a predictor for CSF.

\section{Material and methods Study population}

The study population included 40 patients who had previously undergone angiography in the cardiology clinic of Elaziğ Education and Research Hospital in Elaziğ, Turkey, between January 2013 and December 2014 and who were found to have normal coronary anatomy (NCA), and 40 patients diagnosed with CSF, which was calculated by thrombolysis in myocardial infarction (TIMI) square. A total of 80 patients were included in the study retrospectively and all laboratory and anamnesis information was scanned into their files. The medical records used contained anonymous and de-identified data. The relationship between MPV and CSF was investigated. The study was initiated after obtaining approval of the ethical committee (Presidency of T.C. Firat University Ethics Committee) and was conducted under the regulations determined by the Declaration of Helsinki. The committee did not require that written informed consent be obtained, due to the anonymous nature of the data.

\section{Laboratory measurements}

Blood samples ( $6 \mathrm{~mL}$ for full biochemistry, $5 \mathrm{~mL}$ for complete blood count) were obtained from the antecubital vein prior to CAG and following a 12 hour fast. Samples were drawn into vacuum tubes containing 15\% K3 ethylene diamine tetraacetic acid (EDTA)-anticoagulation tubes (Sarstedt, Essen, Belgium) and analyzed. Hematocrit, hemoglobin, platelets, MPV, and white blood cells (WBCs) were assessed using a Sysmex SE 9500 hematology analyzer (Roche Diagnostics, Mannheim, Germany) according to the manufacturer's instructions. MPV was evaluated using a 3 $\mathrm{mL}$ sample of venous blood collected in EDTA-containing anticoagulation tubes. We analyzed the blood samples of all of the groups using an automatic blood counter after half an hour of venipuncture. The half hour waiting period was allowed for the stabilization of platelet shape changes. Glucose, urea, creatinine, total cholesterol, triglycerides, highdensity lipoprotein, and low-density lipoprotein (LDL) levels were measured using a chemiluminescence method with a Cobas e-601 autoanalyzer and associated reagents (Roche Diagnostics) according to the manufacturer's instructions.

\section{CAG and assessment of CSF}

CAG was performed using the Judkins technique with a Siemens Axiom Artis FC diagnostic device (Siemens Healthineers, Forchheim, Germany). ${ }^{18} \mathrm{CAG}$ equipment was used and left heart catheterization and right and left coronary angiographies were conducted. Left anterior oblique and cranial, right anterior oblique and caudal and horizontal position CAG records were obtained. During the coronary angiogram, iohexol $350 \mathrm{mg}$ iodine/mL (Amersham Health, Cork, Ireland) was used as the opaque substance. During the procedure, at least $6 \mathrm{~mL}$ of the opaque substance was injected into coronaries at each position.

\section{CSF and TIMI frame number}

There are two methods of quantifying "slow flow." The first is TIMI flow grade, which scores flow level from TIMI 0 (no flow) to TIMI 3 (normal flow; distal vessel is opacified in $\leq 3$ beats). TIMI 1 flow (penetration without perfusion) is faint antegrade coronary flow beyond the occlusion with incomplete filling of the distal coronary bed. TIMI 2 flow (partial reperfusion) is delayed or sluggish antegrade flow with complete filling of the distal territory. A TIMI 2 flow grade (requiring $\geq 3$ beats to opacify the vessel) has been 
frequently used to diagnose CSF. The second method is the corrected TIMI frame count, which is the number of frames required for the contrast material to reach a specified distal coronary artery point. ${ }^{2,19,20}$ A corrected TIMI frame count $>27$ frames has been frequently used. The second method is based on images acquired at 30 frames/second, with a correction factor of 1.7 for the left anterior descending (LAD) artery. $^{2}$ Here, the TIMI frame count method was used to identify patients with CSF. To objectively evaluate coronary blood flow as a numeric variable, the required number of sine frames for the contrast substance to reach the distal end of the LAD artery was accepted as the TIMI frame count. TIMI frame count was calculated on a Siemens Axiom Artis FC diagnostic device (Siemens Healthineers) using Scientific Quantification Coronary Analysis software (Siemens Healthcare Gmbh, Forcheim, Germany). Second CAG images obtained 36-48 hours after the initial diagnosis were used when calculating TIMI frame count and the average of measurements obtained in at least three positions was used. During measurement, the first frame where the contrast substance entered the artery was accepted as the first frame. The frame in which the contrast substance reached the distal end of the LAD coronary artery was accepted as the last frame. Then, the difference between the first and last frames was measured to obtain the TIMI frame count. ${ }^{21}$ Angiographic assessments were conducted by at least two experienced angiography specialists. For opaque substance measurement and determination of the CSF sample, the TIMI frame count method was used. ${ }^{22}$ The frame where the opaque substance was introduced to the coronary artery ostium and the coronary artery was visible was accepted as the first frame in which the opaque substance fully enters the artery. This occurs when three criteria are met: 1) a column of nearly full or fully concentrated dye must extend across the entire width of the origin of the artery; 2) the dye must touch both borders of the origin of the artery; and 3) there must be antegrade motion to the dye. The last frame is counted or included as one of the frames and is defined as the frame when the dye first enters the distal landmark branch. Full opacification of the branch is not required. Often, the last frame is best determined by running the cine film past the initial opacification of the endpoint branch and then moving frame-by-frame in reverse until the endpoint branch disappears. To identify the frame in which the dye first appears, care must be taken to advance one frame forward once the dye disappears. The distal point is accepted as the last frame. Distal bifurcation for the LAD artery (known as "mustache," "pitchfork," or "whale's tail"), end of distal bifurcation for circumflex (Cx) artery, and the first side branch of the posterolateral artery for the right coronary artery (RCA) were accepted as distal points. The difference between the first and last frames was accepted as the frame count. Since the distance between proximal and distal bifurcation in the LAD artery is longer than other coronary arteries, LAD TIMI frame count is significantly higher than RCA and Cx TIMI frame counts. Thus, Gibson et $\mathrm{al}^{22}$ divided LAD frame count by the average frame counts obtained from $\mathrm{Cx}$ and RCA and found 1.7 constant coefficients to standardize the measurements. As normal frame counts are necessary to fill the coronary arteries and corrected for coronary artery length, mean reference values of $36 \pm 1$ for LAD, 22.2 \pm 4 for Cx, and 20.4 \pm 3 for RCA were obtained by Gibson. In the present study, standard two units above these reference values were accepted, and $>38$ for $\mathrm{LAD},>30$ for $\mathrm{Cx}$, and $>26$ for RCA were accepted as CSF.

\section{Exclusion criteria}

Patients with idiopathic thrombocytopenic purpura, obesity (body mass index $>30$ ), sepsis, prosthetic heart valves, acute myeloid leukemia, chronic myeloid leukemia, splenectomy, vasculitis, megaloblastic anemia, acute coronary syndrome, presence of significant hemorrhage, chronic kidney disease, thyroid dysfunction, chronic lung disease, malignant arrhythmia, connective tissue disease, heart failure, and cardiomyopathy were excluded from the study.

\section{Statistical evaluation}

Statistical analyses were performed using SPSS software, version 15.0 (SPSS Inc., Chicago, IL, USA) for Windows. The Kolmogorov-Smirnov test was used to evaluate whether the variables were normally distributed. Continuous variables were presented as means with standard deviations or medians with 25 th-75th percentiles. Categorical variables were represented as numbers with percentages. Continuous data were analyzed by Student's $t$-test for normally distributed variables and Mann-Whitney $U$ test for non-normally distributed variables. Categorical data were analyzed using chi-square test or Fisher's exact test. Multivariate regression analysis was performed to determine which clinical variables would independently predict the CSF. CSF was used in the model as a dependent variable. MPV, age, WBC, hematocrit, LDL, glucose, potassium, and creatinine were treated as independent variables. Results were presented as beta coefficients and 95\% confidence intervals (CIs). All $p$-values were two-tailed, and values $<0.05$ were considered to indicate statistical significance. Receiver operating characteristics (ROC) test was used to assess the sensitivity and specificity of MPV and its optimal cutoff value. Level of significance was set at $p<0.05$. 


\section{Results}

There were no statistically significant differences between the CSF and NCA groups based on atherosclerosis risk factor values such as age, gender, HL, smoking, HT, DM, LDL, triglycerides, hemoglobin, hematocrit, glucose, WBCs, Na, urea, creatinine, and $\mathrm{K}$ values (Table 1). It was observed that MPV values for the CSF group were significantly higher compared to the NCA group $(10.05 \pm 1.3$ and $8.6 \pm 0.6, p<0.001)$ (Table 1, Figure 1).

ROC analyses demonstrated that predictive specificity and sensitivity for an MPV >9.05 measured in CSF patients at

Table I Intergroup comparison of demographical data

\begin{tabular}{|c|c|c|c|}
\hline & CSF & Control & $p$-value \\
\hline Gender (male/female) & $20 / 20$ & $19 / 21$ & 0.90 \\
\hline Age (years) & $54.5 \pm 8.7$ & $52.4 \pm 10.6$ & 0.3 \\
\hline Hypertension, n (\%) & $17(42.5)$ & $9(22.5)$ & 0.1 \\
\hline Diabetes mellitus, $\mathrm{n}(\%)$ & $6(15)$ & $3(7.5)$ & 0.5 \\
\hline Smoking, n (\%) & $10(25)$ & $8(20.0)$ & 0.8 \\
\hline Hyperlipidemia, n (\%) & $12(30)$ & $\mathrm{II}(27.5)$ & 1.00 \\
\hline Platelet $\left(10^{3} / \mathrm{mm}^{3}\right)$ & $257 \pm 61$ & $257 \pm 53$ & 1 \\
\hline Mean platelet volume (fL) & $10.0 \pm 1.3$ & $8.6 \pm 0.6$ & $<0.001$ \\
\hline $\begin{array}{l}\text { White blood cell } \\
\left(10^{3} / \mathrm{mm}^{3}\right)\end{array}$ & $7.3 \pm 1.8$ & $6.6 \pm 1.8$ & 0.1 \\
\hline Hematocrit (\%) & $42.3 \pm 4.0$ & $40.8 \pm 3.6$ & 0.07 \\
\hline Hemoglobin $(\mathrm{g} / \mathrm{dL})$ & $14.0(12.9-15.3)$ & $13.9(12.3-14.7)$ & $0.3^{*}$ \\
\hline $\begin{array}{l}\text { Low-density lipoprotein } \\
(\mathrm{mg} / \mathrm{dL})\end{array}$ & $109.4 \pm 30.0$ & $|20| \pm 48.7$. & 0.2 \\
\hline Triglycerides (mg/dL) & $160.0 \pm 8 \mid .2$ & $188.5 \pm 88.7$ & 0.1 \\
\hline Glucose (mg/dL) & $100(92-115)$ & $99(92-112)$ & $0.8^{*}$ \\
\hline Sodium (mmol/L) & $138.8 \pm 3.2$ & $\mid 40.1 \pm 2.7$ & 0.7 \\
\hline Potassium (meq/L) & $4.2 \pm 0.4$ & $4.2 \pm 0.4$ & 0.4 \\
\hline Urea (mg/dL) & $35.5(31.0-43.5)$ & $36(35-39)$ & $0.9^{*}$ \\
\hline Creatinine (mg/dL) & $0.85 \pm 0.20$ & $0.83 \pm 0.16$ & 0.7 \\
\hline
\end{tabular}

Notes: *Mann-Whitney $U$ test. Values shown as $n / n$, mean \pm standard deviation, $n$ (\%), median (interquartile range). Control: normal coronary artery.

Abbreviation: CSF, coronary slow flow.



Figure I Comparison of MPV between CSF and NCA.

Abbreviations: CSF, coronary slow flow; MPV, mean platelet volume; NCA, normal coronary anatomy.

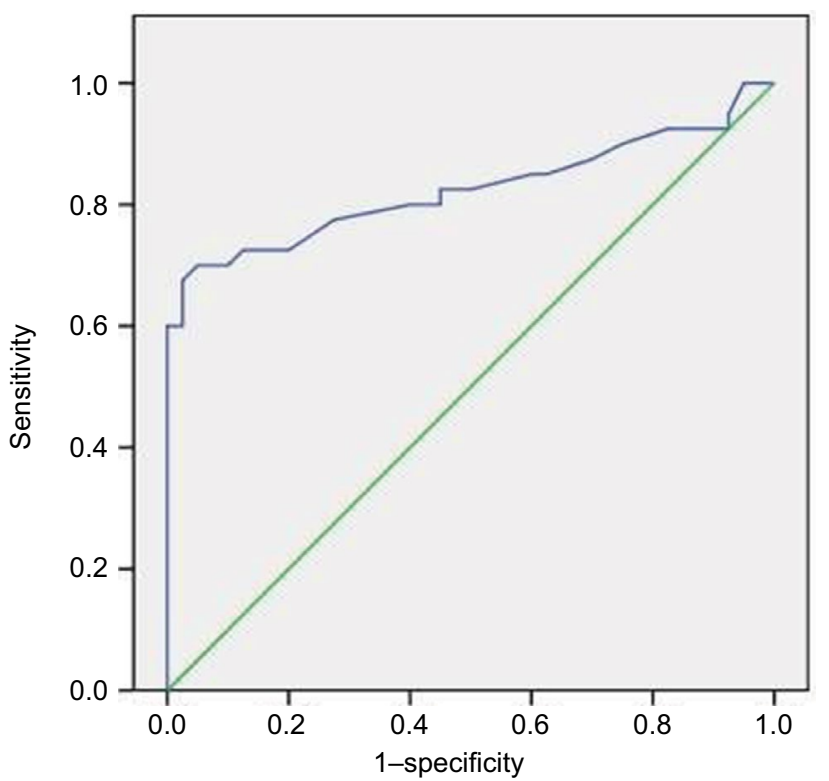

Figure 2 MPV ROC analysis between CSF and NCA groups.

Notes: AUC: $0.825,95 \% \mathrm{Cl}: 0.726-0.924, p<0.000$ I; specificity: 77.5 ; sensitivity: 77.5; cutoff: $>9.05$

Abbreviations: $\mathrm{AUC}$, area under the curve; $\mathrm{Cl}$, confidence interval; CSF, coronary slow flow; MPV, mean platelet volume; NCA, normal coronary anatomy; ROC, receiver operating characteristics.

application were $77.5 \%$ and $77.5 \%$, respectively (area under the curve: 0.825 , 95\% CI: 0.726-0.924, $p<0.0001$ ) (Figure 2).

Multivariate logistic regression analysis results showed that MPV levels, unlike other parameters, were independent predictors of CSF $(\beta=-600, p<0.001,95 \% \mathrm{CI}:-0.383$ to -0.176 ) (Table 2).

\section{Discussion}

The objective of the present study was to investigate whether MPV levels could be an indicator of CSF. The results demonstrated that MPV levels were significantly higher in patients with CSF. In the present study, we found higher MPV values in the CSF group compared to the NCA group $(p<0.001)$ (Table 1, Figure 1). Furthermore, it was shown that MPV levels were independent predictors of CSF $(\beta=-600, p<0.001$, 95\% CI: -0.383 to -0.176 ) (Table 2).

During CAG, the CSF phenomenon is observed as delayed distal vessel opacification in the absence of significant coronary stenosis. The mechanism of this angiographic clinical entity remains unknown, although several hypotheses have been proposed, including inflammation, endothelial dysfunction, changes in blood rheological properties, increased uric acid, and conditions associated with atherosclerotic risk factors. ${ }^{23}$ The cellular components of the blood in patients with CSF have not been evaluated comprehensively.

Platelet function can be affected by several factors, such as platelet count, size, density, platelet age, higher granule 
Table 2 Multivariate logistic regression analysis results for CSF

\begin{tabular}{lllll}
\hline & $\beta$ & $\boldsymbol{p}$-value & \multicolumn{2}{c}{ 95\% Confidence interval } \\
\hline Age & 0.02 & 0.989 & -0.012 & 0.012 \\
MPV & -0.600 & $<0.001$ & -0.383 & -0.176 \\
WBC & -0.051 & 0.63 & -0.075 & 0.046 \\
Htc & -0.182 & 0.011 & -0.054 & 0.006 \\
LDL & 0.023 & 0.84 & -0.002 & 0.003 \\
Glucose & 0.051 & 0.63 & -0.004 & 0.006 \\
K & -0.004 & 0.96 & -0.267 & 0.278 \\
Urea & 0.128 & 0.24 & -0.006 & 0.022 \\
\hline
\end{tabular}

Abbreviations: CSF, coronary slow flow; Htc, high total cholesterol; K, potassium LDL, low-density lipoprotein; MPV, mean platelet volume; WBC, white blood cell.

count, and adhesion receptor expression, as well as a higher MPV. MPV appears to correlate more closely with platelet function than does platelet count alone. ${ }^{24}$ In previous reports, the following methods were used to analyze platelet activation: optical aggregometry, platelet function analysis using a PFA-100 system, platelet reactivity tests, platelet aggregate ratio measurements, flow cytometry, and thromboxane B2 generation tests. ${ }^{25}$ All of these tests are of limited use due to complex pre-analytic factors, reduced specificity, and poor reproducibility. MPV is a simple marker that does not require advanced or expensive technology. However, it may reflect platelet function and activity and may indirectly reflect platelet production and stimulation; therefore, elevated MPV values may indicate cardiovascular diseases. ${ }^{17}$

High MPV levels were reported in studies conducted in individuals with coronary heart disease. ${ }^{26}$ In addition to endothelial adhesion and aggregation, platelet activation in turn activates the inflammatory process by interacting with WBCs. ${ }^{27}$ Platelet activation and platelet-WBC interaction play significant roles in atherothrombotic diseases. ${ }^{28}$ Platelets also play an important role in the pathogenesis of atherosclerotic complications and thrombus formation. Platelet size measured as MPV is a good indicator of specific platelet activation such as platelet aggregation, TxA2, platelet factor 4 , and thromboglobulin release. ${ }^{29,30}$ Platelet volume is determined by megakaryocytes in thrombosis because large platelets are created by the disintegration of large megakaryocytes. Several studies claim that there is a correlation between platelet volumes and functions. There are also publications reporting that platelets with large volumes are more active than those with a small volume and thus could be an indicator of risk for acute coronary syndrome. ${ }^{31}$

Although it is considered that cases with chest pain and CSF generally have a good prognosis, continuation of the symptoms, and possibility of candidacy for acute coronary syndrome, they are not considered totally benign. ${ }^{32-34}$
Although it has been reported that myocardial infarction could develop in cases with CSF, it is still unknown if CSF has an effect that increases thrombosis. ${ }^{35}$ Furthermore, it has been demonstrated that coronary flow could revert to normal with dipyridamole treatment. ${ }^{36}$

In our study, which was conducted in the light of above mentioned information, we found higher MPV values in the CSF group compared to the NCA group $(p<0.001)$ (Table 1 , Figure 1). It was demonstrated that atherosclerosis risk factors were correlated with increase in MPV. ${ }^{37,38}$ In the present study, although HT, DM, smoking, and average age were higher in the CSF group than in the control group, patient populations in both groups were statistically similar with respect to these risk factors. On the other hand, triglycerides levels were moderately elevated in both groups, and the two groups did not differ with respect to triglycerides levels or HL risk. This situation may be attributed to the limited number of patients, genetic features, or eating habits of our region's people. Higher MPV values in the CSF group observed in this study support the premise that high MPV values could indicate atherosclerotic heart diseases. ${ }^{26}$ It has been suggested that endothelial and microvascular dysfunctions are responsible for CSF.,3 Histopathologic studies have demonstrated that the inflammatory process has an important role in CSF. $^{4,39}$ In addition, it must be remembered that platelet function disorder is one of the reasons behind $\mathrm{CSF}^{40} \mathrm{CSF}$ pattern causes thrombus and therefore distal embolism or myocardial infarction may occur. ${ }^{32,34,35}$ High MPV levels have been reported in connection with acute coronary syndrome as well. ${ }^{31}$ The high MPV levels observed in this study might support the suggestion that thrombus formation, distal embolism, and acute vascular events may occur in CSF. Finally, our study might suggest that using antiplatelet treatment in CSF to prevent distal embolism (or microvascular embolism) may be helpful in decreasing chest pain and hospitalization. Further comprehensive and long-term studies are needed to clarify this issue.

\section{Limitations of the study}

There are a few limitations of the present study. Primarily, the study was conducted with a limited number of patients. Secondly, assessment of CSF patients diagnosed with CSF using angiography was carried out visually. However, intravascular ultrasound and autopsy studies in patients diagnosed with CSF evidenced the existence of wide atherosclerotic plaques. Thus, another limitation of the present study was the inability to use intravascular ultrasound. 


\section{Conclusion}

The present study demonstrated that MPV levels in CSF patients were higher than in NCA patients. Furthermore, the study results show that MPV level was an independent predictive parameter in CSF pathogenesis. Thus, antiplatelet treatment may be essential in CSF.

\section{Disclosure}

The authors report no conflicts of interest in this work.

\section{References}

1. Xia S, Deng SB, Wang Y, et al. Clinical analysis of the risk factors of slow coronary flow. Heart Vessels. 2011;26:480-486.

2. Mullasari A, Victor SM. Coronary slow flow phenomenon. Available from: https://www.escardio.org/Journals/E-Journal-of-CardiologyPractice/Volume-11/Coronary-slow-flow-phenomenon. Accessed January 20, 2017.

3. Mangieri E, Macchiarelli G, Ciavolella M, et al. Slow coronary flow: clinical and histopathological features in patients with otherwise normal epicardial coronary arteries. Cathet Cardiovasc Diagn. 1996;37:375-381.

4. Mosseri M, Yarom R, Gotsman MS, Hasin Y. Histologic evidence for small-vessel coronary artery disease in patients with angina pectoris and patent large coronary arteries. Circulation. 1986;74:964-972.

5. Gawaz M, Langer H, May AE. Platelets in inflammation and atherogenesis. J Clin Invest. 2005;115(12):3378-3384.

6. Yaşar AS, Bilen E, Yüksel IO, et al. Association between admission mean platelet volume and coronary patency after thrombolytic therapy for acute myocardial infarction. Turk Kardiyol Dern Ars. 2010;38(2):85-89

7. Balli M, Taşolar H, Çetin M, et al. Relationship of platelet indices with acute stent thrombosis in patients with acute coronary syndrome. Postepy Kardiol Interwencyjnej. 2015;11(3):224-229.

8. Ranjith MP, DivyaRaj R, Mathew D, George B, Krishnan MN. Mean platelet volume and cardiovascular outcomes in acute myocardial infarction. Heart Asia. 2016;8(1):16-20.

9. Thompson CB, Eaton KA, Princiotta SM, Rushin CA, Valeri CR. Size dependent platelet subpopulations: relationship of platelet volume to ultrastructure, enzymatic activity, and function. $\mathrm{Br} J$ Haematol. 1982;50:509-519.

10. Doğan NO, Ayfer K, Aksel G, et al. Mean platelet volume as a risk stratification tool in the emergency department for evaluating patients with ischaemic stroke and TIA. J Pak Med Assoc. 2013;63(5):581-584.

11. Tsiara S, Elisaf M, Jagroop IA, Mikhailidis DP. Platelets as predictors of vascular risk: is there a practical index of platelet activity? Clin Appl Thromb Hemost. 2003;9:177-190.

12. Pal R, Bagarhatta R, Gulati S, Rathore M, Sharma N. Mean platelet volume in patients with acute coronary syndromes: a supportive diagnostic predictor. J Clin Diagn Res. 2014;8(8):1-4.

13. Ulutas KT, Dokuyucu R, Sefil F. Evaluation of mean platelet volume in patients with type 2 diabetes mellitus and blood glucose regulation: a marker for atherosclerosis? Int J Clin Exp Med. 2014;7(4):955-961.

14. Uçar H, Gür M, Gözükara MY. Relationship between mean platelet volume and morning blood pressure surge in newly diagnosed hypertensive patients. Anatol J Cardiol. 2015;15:107-112.

15. Andronescu AM, Delcea C, Enache V, Stamate CS, Dorobanțu M. Mean platelet volume variability in young patients with non-ST elevation acute myocardial infarction. J Med Life. 2014;7(3):107-113.

16. Demir S, Avsar MK, Karakaya Z, et al. Increased mean platelet volume is associated with coronary artery ectasia. Postepy Kardiol Interwencyjnej. 2013;9(3):241-245.

17. Demirkol S, Balta S, Unlu M, et al. Evaluation of the mean platelet volume in patients with cardiac syndrome X. Clinics (Sao Paulo). 2012; 67(9):1019-1022.
18. Fuster V, Walsh R, Harrington R. Cardiac catheterization, cardiac angiography and coronary blood flow and pressure measurements. In: Hurst's the Heart. 12th ed. New York: McGraw-Hill Companies; 2009:471-474.

19. Oktay V, Arat Özkan A. Coronary slow flow. Turk Kardiol Dern Ars. 2016;44(3):193-195.

20. TIMI grade flow - grading of coronary blood flow during coronary angiography. Available from: http://radclass.mudr.org/content/timigrade-flow-grading-coronary-blood-flow-during-coronary-angiography. Accessed January 20, 2017.

21. Tenekecioğlu E, Karaağaç K, Yontar OC, et al. Evaluation of Tp-Te Interval and $\mathrm{Tp}-\mathrm{Te} / \mathrm{QT}$ ratio in patients with coronary slow flow $\mathrm{Tp}-\mathrm{Te} /$ QT ratio and coronary slow flow. Eurasian J Med. 2015;47:104-108.

22. Gibson CM, Cannon CP, Daley WL, et al. TIMI frame count: a quantitative method of assessing coronary artery flow. Circulation. 1996; 93:879-888.

23. Akpinar I, Sayin MR, Gursoy YC, et al. Plateletcrit and red cell distribution width are independent predictors of the slow coronary flow phenomenon. J Cardiol. 2014;63(2):112-118.

24. Thompson CB, Jakubowski JA. The pathophysiology and clinical relevance of platelet heterogeneity. Blood. 1988;72(1):1-8.

25. Haubelt H, Simon M, Anders C, Hellstern P. Plättchenfunktionstests zum monitoring der azetylsalizylsäuretherapie klinische wertigkeit bei plättchenfunktionshemmender behandlung [Platelet function tests for monitoring of acetylsalicylic acid: clinical significance in antiplatelet treatment]. Hamostaseologie. 2004;24(3):196-202. German.

26. Tamer D, Fevzi Y, Deniz AE, et al. The value of serum mean platelet volume in ischaemic stroke patient. J Pak Med Assoc. 2013;63:1509-1510.

27. Totani L, Evangelista V. Platelet-leukocyte interactions in cardiovascular disease and beyond. Arterioscler Thromb Vasc Biol. 2010;30:2357-2361.

28. Nagy B Jr, Miszti-Blasius K, Kerenyi A, Clemetson KJ, Kappelmayer J. Potential therapeutic targeting of platelet-mediated cellular interactions in atherosclerosis and inflammation. Curr Med Chem. 2012; 19:518-531.

29. Bath PM, Butterworth RJ. Platelet size: measurement, physiology and vascular disease. Blood Coagul Fibrinolysis. 1996;7:157-161.

30. Sharp DS, Benowitz NL, Bath PM, Martin JF, Beswick AD, Elwood PC. Cigarette smoking sensitizes and desensitizes impedance-measured ADP-induced platelet aggregation in whole blood. Thromb Haemost. 1995;74:730-735.

31. Kristensen DS, Martin JF. Megakaryocytes and atherosclerosis. Clin Sci. 1992;82:353-355.

32. Chaudhry MA, Smith M, Hanna EB, Lazzara R. Long-term clinical course of patients with angina and angiographically normal coronary arteries. Cardiol Res Pract. 2012;2012:1-5.

33. Amasyali B, Turhan H, Kose S, et al. Aborted sudden cardiac death in a 20-year-old man with slow coronary flow. Int J Cardiol. 2006; 109(3):427-429.

34. Beltrame JF, Limaye SB, Horowitz JD. The coronary slow flow phenomenon - a new coronary microvascular disorder. Cardiology. 2002; 97:197-202.

35. Oz F, Çizgici AY, Kaya MG, et al. Low bilirubin levels are associated with coronary slow flow phenomenon. Kardiol Pol. 2015;73(1):40-45.

36. Kurtoğlu N, Akçay A, Dindar I. Usefulness of oral dipyridamole therapy for angiographic slow coronary artery flow. Am J Cardiol. 2001;87:777-779.

37. Vizioli L, Muscari S, Muscari A. The relationship of mean platelet volume with the risk and prognosis of cardiovascular diseases. Int $J$ Clin Pract. 2009;63:1509-1515.

38. Keskin S, Gürler M, Temeloğlu E, Çelebi A, Alicanoğlu R, Ekizoğlu İ. Relation between mean platelet volume and risk factors for coronary heart disease. J Med Sci. 2006;26(4):380-384.

39. Demir M, Demir C, Keçeoğlu S, Aktaş I. Evaluation of plasma monocyte count in patients with coronary slow flow. Acta Medica Mediterr. 2015;31:705-709.

40. Demir M, Coşar S, Melek M. Evaluation of plasma eosinophil count and mean platelet volume in patients with coronary slow flow. Clinics (Sao Paulo). 2014;69(5):323-326. 


\section{Publish your work in this journal}

Vascular Health and Risk Management is an international, peerreviewed journal of therapeutics and risk management, focusing on concise rapid reporting of clinical studies on the processes involved in the maintenance of vascular health; the monitoring, prevention and treatment of vascular disease and its sequelae; and the involvement of metabolic disorders, particularly diabetes. This journal is indexed on PubMed Central and MedLine. The manuscript management system is completely online and includes a very quick and fair peer-review system, which is all easy to use. Visit http://www.dovepress.com/ testimonials.php to read real quotes from published authors.

Submit your manuscript here: https://www.dovepress.com/vascular-health-and-risk-management-journal 